\title{
Hybrid video-assisted thoracoscopic surgery sleeve lobectomy for non-small cell lung cancer: a case report
}

\author{
Chenlei Zhang ${ }^{1}$, Zhanwu Yu ${ }^{1}$, Jijia Li ${ }^{1}$, Peng Zu ${ }^{1}$, Pingwen $\mathrm{Yu}^{1}$, Gebang Wang ${ }^{1}$, Takuro Miyazaki ${ }^{2}$, \\ Ryuichi Waseda ${ }^{3}$, Raul Caso ${ }^{4}$, Giulio Maurizi ${ }^{5}$, Hongxu Liu ${ }^{1}$ \\ ${ }^{1}$ Department of Thoracic Surgery, Cancer Hospital of China Medical University, Liaoning Cancer Hospital \& Institute, Shenyang, China; \\ ${ }^{2}$ Department of Surgical Oncology, Nagasaki University Graduate School of Biomedical Sciences, Nagasaki, Japan; ${ }^{3}$ Department of General \\ Thoracic, Breast, and Pediatric Surgery, Fukuoka University, Fukuoka, Japan; ${ }^{4}$ Department of Surgery, MedStar Georgetown University Hospital, \\ Washington, DC, USA; ${ }^{5}$ Department of Thoracic Surgery, Sant'Andrea Hospital, Sapienza University of Rome, Rome, Italy \\ Correspondence to: Hongxu Liu. Department of Thoracic Surgery, Cancer Hospital of China Medical University, Liaoning Cancer Hospital \& \\ Institute, No.44 Xiaoheyan Road, Dadong District, Shenyang 110042, China. Email: hongxuliu@qq.com.
}

Submitted Feb 04, 2020. Accepted for publication Oct 24, 2020.

doi: $10.21037 /$ jtd-20-2679

View this article at: http://dx.doi.org/10.21037/jtd-20-2679

\section{Introduction}

Lung cancer continues to be the leading cause of cancerrelated death in China and worldwide $(1,2)$. Sleeve lobectomy (SL) and pneumonectomy are surgical options for the treatment of locally advanced non-small cell lung cancer (NSCLC). SL was initially intended as a parenchymasparing treatment for patients who were unable to tolerate pneumonectomy, but now it is a standard approach for the treatment of anatomically suitable tumors (3). SL offers several advantages such as better overall survival, disease-free survival, postoperative pulmonary function, and quality of life over pneumonectomy (4-7).

Sleeve lobectomies and double sleeve lobectomies are complicated procedures, even when performed with an open approach. The complexity of the procedure makes SL a technically challenging operation, and it was considered a contraindication for video-assisted thoracoscopic surgery (VATS) in many institutions $(8,9)$. It is challenging to perform SL via VATS for most surgeons, especially double sleeve resection. Reports in the literature are limited, and even surgeons performing VATS in their daily practice have been slow to adopt and develop this technique (10-12). A complete VATS approach can be challenging even for experienced VATS surgeons. Here, we describe a less invasive hybrid VATS approach, which lies between open and VATS SL. A hybrid VATS approach allows the surgeon to have direct access to the tumor, ensure a safe surgical margin, and save some time during the hybrid VATS procedure. Also, hybrid VATS SL is a safer approach, especially in patients with infiltration of the pulmonary artery (PA), as it makes the surgeon more comfortable during the bronchial and arterial anastomoses. Literatures have shown that hybrid VATS approach has advantages for select T3 chest wall lung cancers (13) and superior sulcus tumors (14) over conventional open surgery. Dr. He and his colleagues (15) reported that hybrid VATS sleeve lobectomy is feasible for selected patients with NSCLC after long-term follow-up period. Here we provide an intraoperative video of a hybrid VATS SL to demonstrate the technical details of the surgical procedure. We present the following case in accordance with the CARE reporting checklist (available at http://dx.doi.org/10.21037/jtd-20-2679).

\section{Case presentation}

A 49-year-old male patient was admitted to our hospital with cough and hemoptysis, and he had no history of pulmonary or cardiac disease. Computed tomography (CT) evaluation showed a mass at the origin of the right upper lobe bronchus (Figure 1A). The bronchoscopy confirmed the mass at the origin of the right upper lobe bronchus involving the right main bronchus (Figure $1 B$ ). The biopsy result was pulmonary adenocarcinoma. Because of the N2 disease, the patient received two cycles of preoperative chemotherapy with pemetrexed and cisplatin, followed by surgery. The tumor disappeared and was evaluated as a complete 

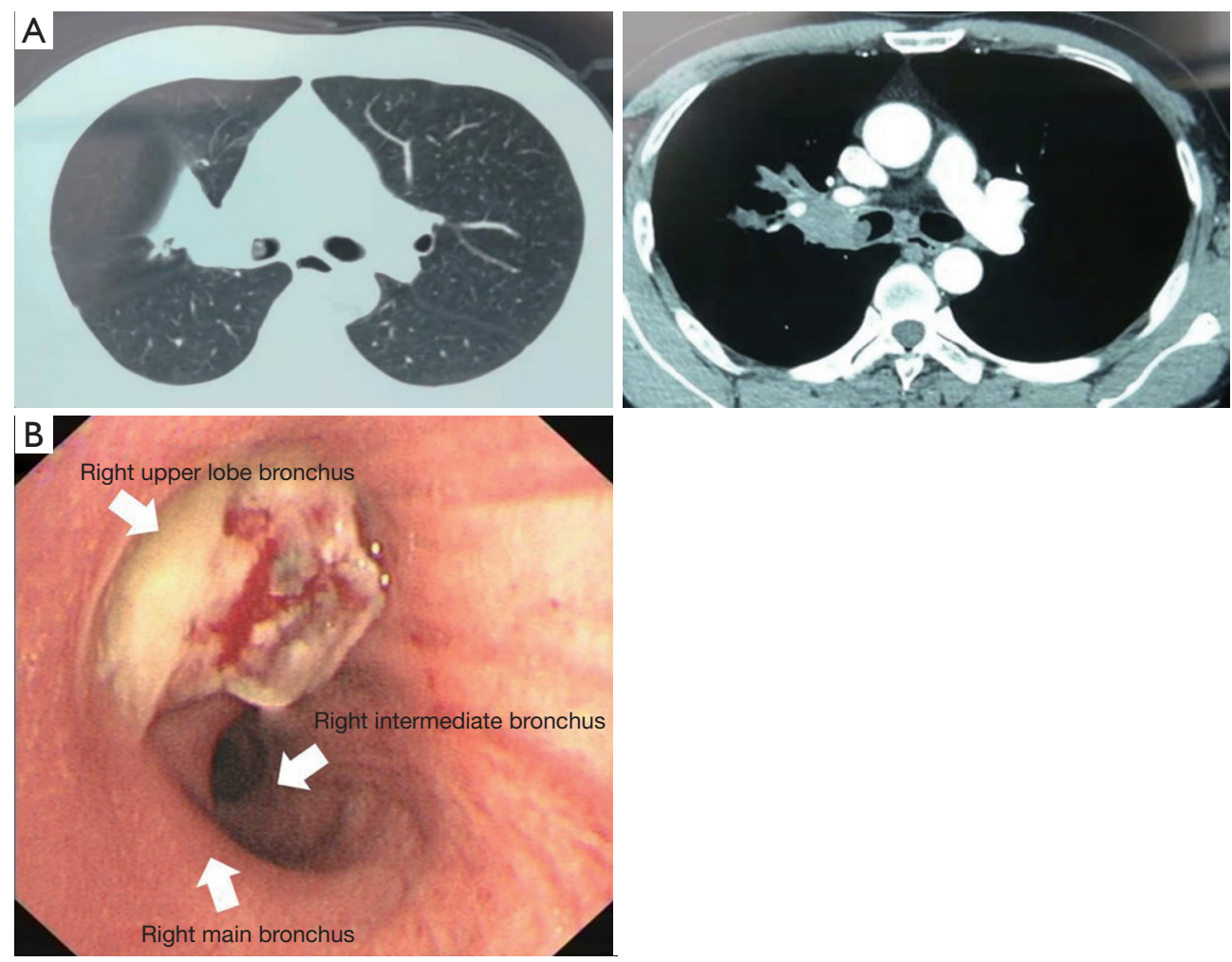

Figure 1 Contrast chest computed tomography and bronchoscopy before neoadjuvant therapy. (A) Chest CT showed the tumor was in the right upper lobe bronchus involving the right main bronchus; (B) bronchoscopy showed the neoplasm found in the opening of the right upper lobe bronchus.

response (CR) after neoadjuvant therapy (Figure 2). A decision was made to perform a hybrid VATS SL.

The patient received intravenous and inhalation anesthesia through double-lumen endotracheal intubation. The patient was placed in a left lateral decubitus position (Figure $3 A$ ). The surgeon stood on the ventral side of the patient, while the first and second assistants stood on the dorsal part of the patient. An $8 \mathrm{~cm}$-long hybrid VATS incision was made in the $5^{\text {th }}$ intercostal space, and an incision protection retractor was used without rib spreader (Figure $3 B$ ). A $1 \mathrm{~cm}$ camera port was placed in the posterior axillary line of $8^{\text {th }}$ intercostal space.

The concept of the hybrid VATS approach can be summarized as combining advantages of $2 \mathrm{D}$ screen view from VATS and direct views from conventional thoracotomy. By looking at the screen, surgeons can carry out most of the procedures, while for performing the anastomosis, direct visualization through a small incision is of great benefit. The $8 \mathrm{~cm}$-long incision is usually enough to supply direct access and evaluation of the tumor and offers an adequate operative field and view. This hybrid approach also ensures direct manipulation during the whole procedure and saves time for bronchial or arterial anastomosis. Room-fixed and thoracoscope light are simultaneously used to increase the brightness of the operative field, and we strongly recommend that a head-mounted light is used to compensate for the limited operative view. The vascular and bronchial structures can be easily accessed using the hybrid VATS incision, and it is easier for the bronchial or arterial anastomosis.

The process of the resection was as follows (Video 1):

(I) First, we opened the oblique and horizontal fissure with a combination of blunt and sharp dissection. The right hilum was opened and mobilized, and lymph nodes of station 10, 2, and 4 were dissected (Figure 4).

(II) The right superior PA (A1 + recurrent A2 + A3b) was transected with a vascular stapler. We exposed the right upper lobe vein and divided it with a vascular stapler. Then we dissected the ascending 

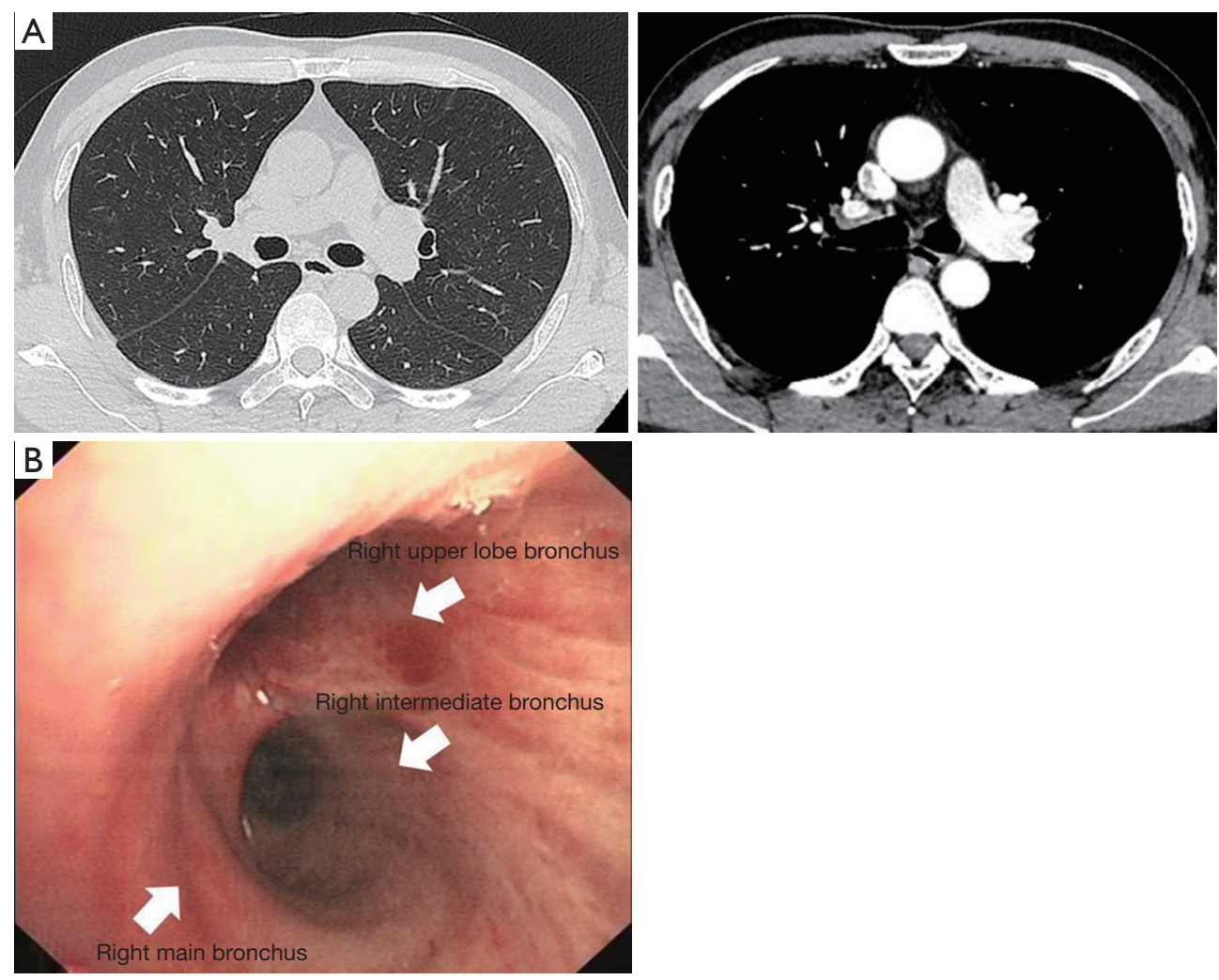

Figure 2 Contrast chest computed tomography (CT) and bronchoscopy after neoadjuvant therapy. (A) Chest CT showed the tumor disappeared after neoadjuvant therapy; (B) bronchoscopy showed the neoplasm disappeared after neoadjuvant therapy.
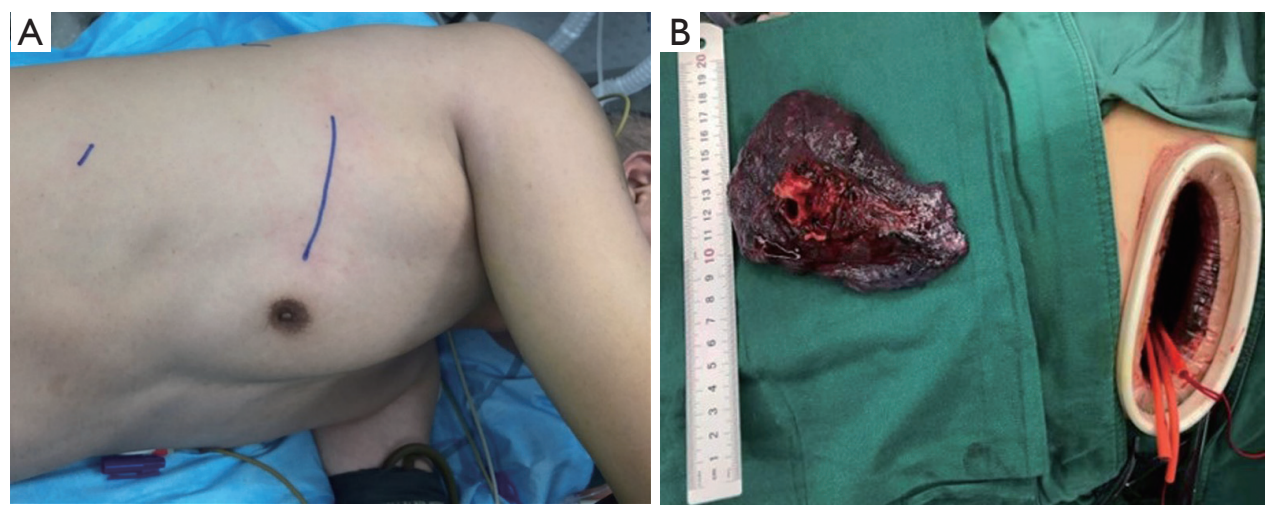

Figure 3 Surgical position and incisions. (A) Patient position and distribution of incisions; (B) an 8 cm hybrid VATS incision was made in the $5^{\text {th }}$ intercostal space, and an incision protection retractor was used without rib spreading.

A2 and A3a and found the tumor and N1 lymph nodes infiltrated the ascending $\mathrm{A} 2$ and $\mathrm{A} 3 \mathrm{a}$. It was challenging to staple the ascending A2 and A3a and ensure a safe surgical margin. Therefore, we dissected and blocked the right intermediate PA, the middle PA, and the lower PA by tapes after systemic heparinization (100 IU/kg, 6,250 IU). Dividing the ascending A2 and A3a with scissors and a direct closure was performed using a running suture with 5-0 prolene (Figure 5). Once the suture 
was completed, we released the tape. The tape would not be entirely removed until we confirmed that there was no bleeding.

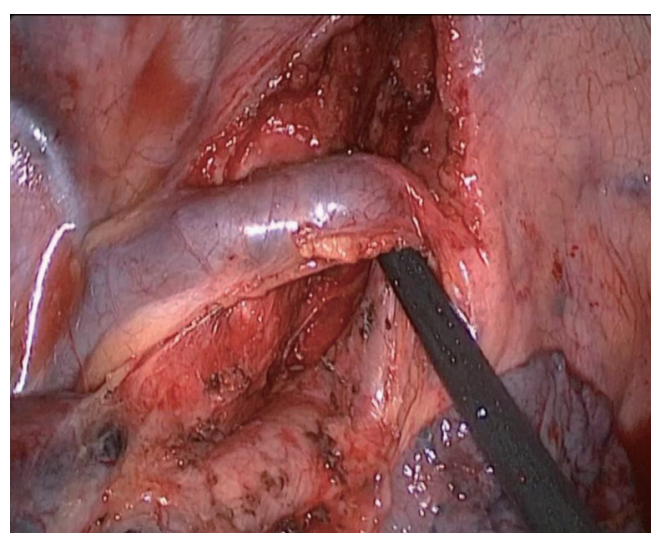

Figure 4 System lymph node dissection: Station 10, 2 and 4 lymph nodes were removed.
(III) We divided the inferior pulmonary ligament and removed the station nine lymph nodes. The posterior mediastinal pleura was incised, and the station seven lymph nodes were removed (Figure 6). Afterward the incomplete fissure was divided with an endostapler, and the distal right main bronchus and bronchus intermedius were fully mobilized to ensure adequate length and reduce tension on the anastomosis. The right main bronchus and intermediate bronchus were sharply divided with a scalpel, and the right upper lobe was removed using an endobag. Intraoperative frozen sections of the bronchial margins from both ends of the right main bronchus and the bronchus intermedius were negative for tumor infiltration. The end-toend anastomosis of the right main bronchus to the intermediate bronchus was performed using the twin-needle stitch and bi-directional continuous full-thickness 4-0 prolene suture (Figure 7). The
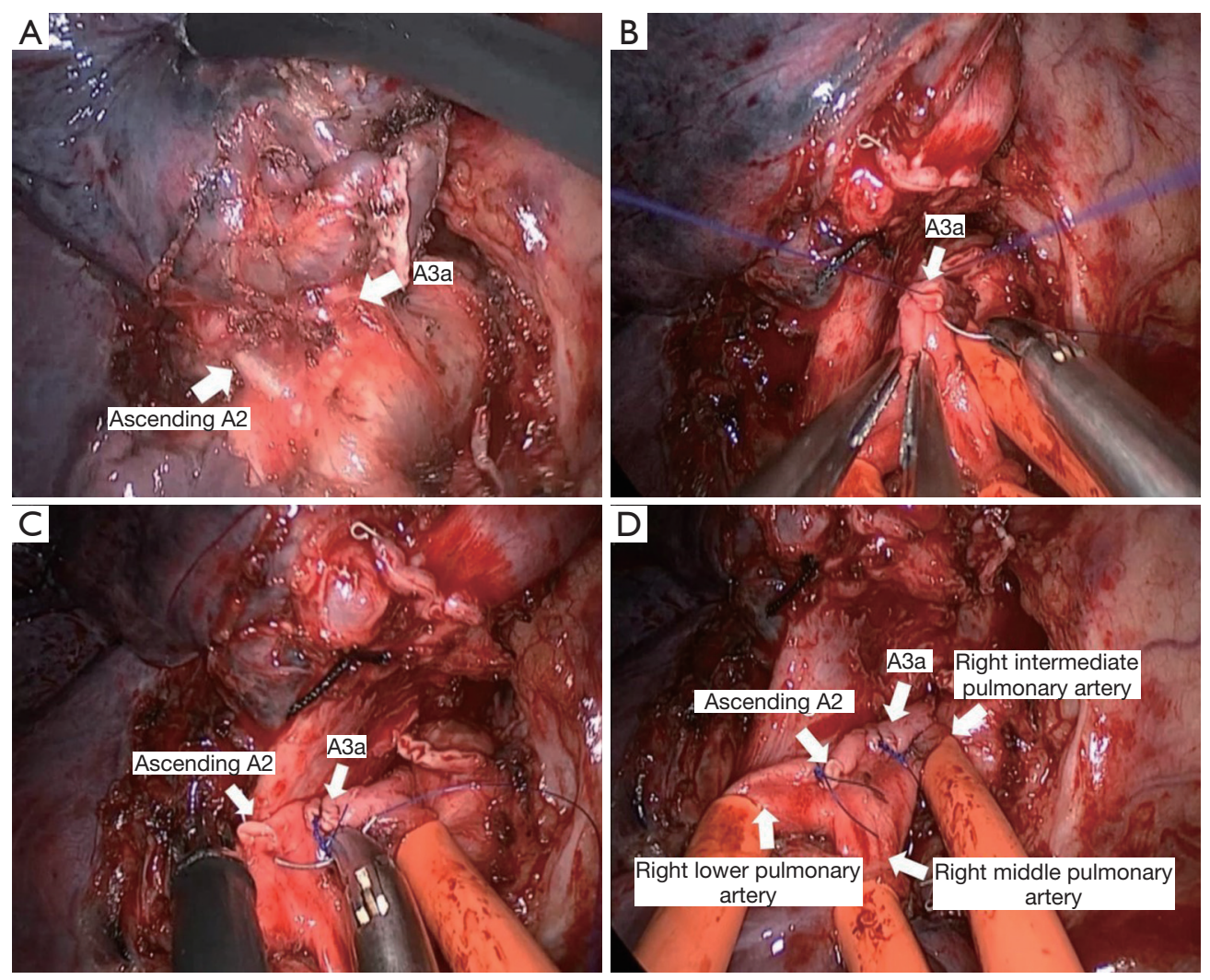

Figure 5 Pulmonary angioplasty. (A) The neoplasm and enlarged lymph nodes infiltrated the pulmonary arteries. (the ascending A2 and A3a). (B,C,D) The right intermediate pulmonary artery, the middle pulmonary artery, and the lower pulmonary artery were blocked by tapes, and the pulmonary arteries were closed using running 5-0 prolene suture. 
water-seal test was conducted following the anastomosis, in which no air leakage was detected up to an airway pressure of $25 \mathrm{cmH}_{2} \mathrm{O}$.

An interrupted suture can reduce the rotational deformity of the bronchial anastomosis (11). However, we are in favor of a continuous suture for multiple advantages over an interrupted suture. It can reduce the time and supply a good angle for anastomosis, and no added sutures are needed. The bronchus anastomotic suturing starts from the posterior wall to the anterior wall. The first bite begins from the cartilaginous part to the membranous part of the bronchus by running full-thickness 4-0 prolene suture. We have used this running suture technique for 135 sleeve resection patients in our institute, and there is no case with bronchial fistula. The patient had a quick recovery without complications and the final pathological result showed that the tumor was evaluated as mCR.

Written informed consent was obtained from the patient for publication of this manuscript and any accompanying images. All procedures performed in studies involving human participants were in accordance with the ethical standards of the institutional and/or national research committee(s) and with the Helsinki Declaration (as revised in 2013).

\section{iMDT}

Question 1: What are the advantages of hybrid videoassisted thoracoscopic surgery sleeve lobectomy for nonsmall cell lung cancer?

\section{Expert opinion 1: Dr. Takuro Miyazaki}

I believe the benefits of hybrid video-assisted thoracoscopic surgery (hybrid VATS) are reduced postoperative pain, earlier recovery of the patients, and earlier discharge from the hospital as authors have already pointed out. I think most thoracic surgeons would agree with the advantages of hybrid VATS compared to standard thoracotomy.

\section{Expert opinion 2: Dr. Ryuichi}

The hybrid approach has 2 big advantages compared with complete VATS as the authors describe in the manuscript.

At first, it allows to perform procedure under direct vision. Although somebody may claim thoracoscopy has better magnified view than direct vision, the direct vision has of course natural 3D view with wide visual field, and even enough magnified view with magnified glasses.

Secondly, an $8 \mathrm{~cm}$ access incision is much more helpful

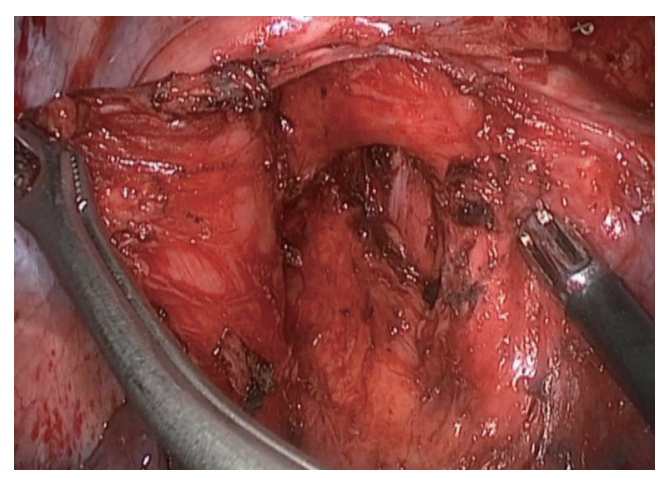

Figure 6 System lymph node dissection: Station 7 lymph nodes were removed.

in manipulations of anastomosis such as needle handling for an operator and suture guiding for an assistant than thoracoscopic ports. The guide of suture means adjusting tension or direction of a suture during anastomosis by an assistant. I think the guide of suture is one of the most important factors to make anastomoses quickly and properly in a limited surgical field.

Those two advantages make the anastomotic procedure simple and safe.

\section{Expert opinion 3: Dr. Raul Caso}

Technical advantages of a hybrid VATS approach to sleeve lobectomy, or in general for any pulmonary resection, include dual visualization, a double light source for the operative field, flexibility in instrumentation, and ability to use the utility incision to gain exposure in control of hemorrhage. It is worth noting, these technical advantages can allow surgeons in community-based practice, where resources, operating room staff, or trained assistants might be limited, to adopt this approach. Utilization of a complete VATS approach can also be limited by the perceived complexity of the technique and concerns regarding the potential compromise of surgical and oncologic principles. A hybrid VATS approach for complex cases, such as a sleeve lobectomy, incorporates some of the advantages of a complete VATS approach with some of the technical advantages of an open approach. Ultimately, this increases the proportion of such complex cases performed by VATS, allowing a greater number of patients to benefit from minimally invasive thoracic surgery.

\section{Expert opinion 4: Dr. Giulio Maurizi}

In my opinion, this approach does not provide any 

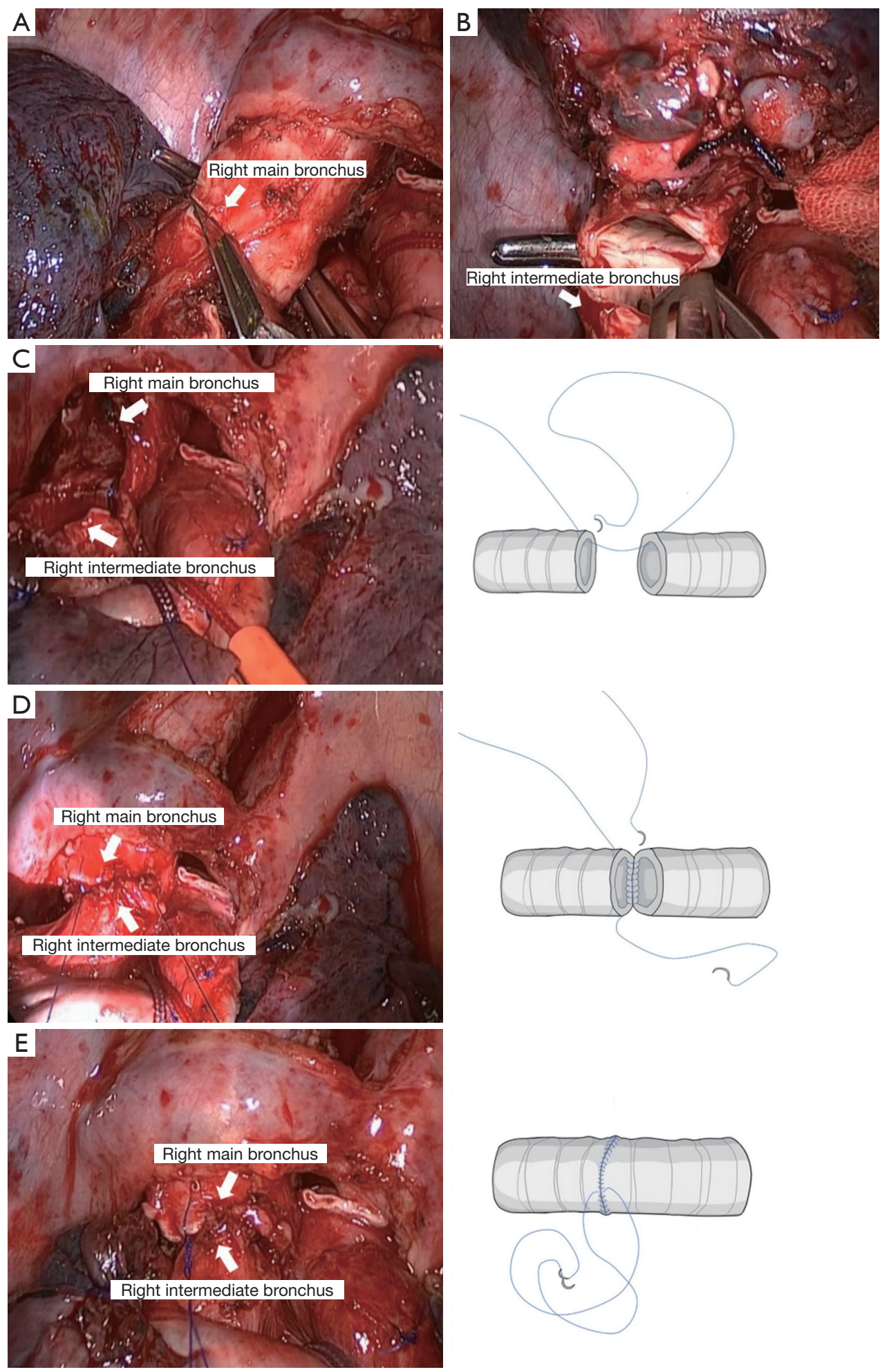

Figure 7 Bronchial sleeve lobectomy. (A) The right main bronchus was divided with a scalpel; (B) the right intermediate bronchus was divided with a scalpel; $(\mathrm{C}, \mathrm{D}, \mathrm{E})$ the end-to-end anastomosis of the right main bronchus to the right intermediate bronchus was performed using the running 4-0 prolene suture. 
advantage. The first reason is that surgeons can already perform a safe sleeve lobectomy by a lateral minithoracotomy or conversely by a full vats approach. Moreover, the approach the authors present in this paper combines vats with a thoracotomy that is not much different from the usual minithoracotomy that is currently employed for open sleeve lobectomy in high-volume thoracic surgery divisions.

\section{Question 2: What are the indications for bybrid video- assisted thoracoscopic surgery sleeve lobectomy for non- small cell lung cancer?}

\section{Expert opinion 1: Dr. Takuro Miyazaki \\ Depending on several factors including intrathoracic adhesions, the size of the tumor, the state of lymph node metastasis, and the skill and experience of the surgeon, hybrid VATS would be firstly attempted if technically possible. However, I have no personal experience with it.}

\section{Expert opinion 2: Dr. Ryuichi}

I think most cases of isolated bronchial sleeve lobectomy can be performed via the hybrid approach. I have not performed broncho-vascular sleeve lobectomy via the hybrid approach. Usually I use small $12-15 \mathrm{~cm}$ thoracotomy with rib spreader for double sleeve procedure. I prefer vascular clamps to tourniquet with tapes to control blood flow of pulmonary artery. If two vascular clamps are used in a $8 \mathrm{~cm}$ incision, surgical field must be congestive. Therefore, I would like to make a bigger incision for double sleeve lobectomy. But I guess hybrid double sleeve procedure may not be so complicated if a vascular clamp is used only for proximal control of PA. I would like to try the procedure in the near future.

\section{Expert opinion 3: Dr. Raul Caso}

A hybrid VATS approach for sleeve lobectomy should be considered by surgeons without sufficient technical experience to perform a completely minimally invasive sleeve lobectomy but still want to adopt the combined visual and technical advantages of a VATS approach. Dual vision allows the surgeon the magnified view from a lower angle with the thoracoscope to be supplemented by the higher angle of vision directly through the utility incision. This gives the surgeon confidence in performing an adequate oncologic resection. Furthermore, when there is PA involvement and therefore concern for bleeding, a hybrid VATS approach allows the surgeon to use the utility incision for immediate access for urgent control of bleeding if necessary which may be challenging by a conventional thoracoscopic approach.

\section{Expert opinion 4: Dr. Giulio Maurizi}

When talking about sleeve lobectomy it would be better to refer not to a single topic but to the different sleeves (upper, lower, middle etc.). Actually, a bronchial carcinoid tumor at the origin of an upper lobe can be easily resected by a full vats upper sleeve lobectomy. Differently, in my opinion, a lung cancer infiltrating the main broncus (in particular the origin of a lower lobe) should be approached by mini-thoracotomy. An open approach may offer a more clear vision of the operative field during the dissection, that could be quite difficult in this setting, and a more precise placement of the stiches when performing the anastomosis, in particular if a large caliber discrepancy between the two bronchial stamps is present.

\section{Question 3: Which method is more appropriate for bronchial anastomosis and pulmonary arterial anastomosis, running suture or interrupted sutures?}

\section{Expert opinion 1: Dr. Takuro Miyazaki}

Our standard fashion for bronchoplasty and/or angioplasty was that the bronchial anastomosis was done using an interrupted suture technique with monofilament absorbable sutures, and were wrapped with a pericardial fat pad or thymus. Suturing of the pulmonary artery was done using a running suture technique with monofilament nonabsorbable sutures. However, recently for bronchoplasty, we perform a running suture for the membranous portion and an interrupted suture for the cartilage portion and, of course, perform a running suture circumferentially because we believe that there is no fundamental difference. Thus, it is hard to say which procedure is superior or inferior, so it may be a matter of the facility's policy, type of thoracotomy or the surgeon's preference.

\section{Expert opinion 2: Dr. Ryuichi}

I have performed both bronchial and arterial anastomosis with a running fashion. 4-0 absorbable monofilament suture is used for bronchial anastomosis and 5-0 non-absorbable monofilament suture for arterial anastomosis. A running fashion is easy and time saving when manipulations are performed in small surgical field. As I describe in Question 1 , the guide of suture by an assistant is quite important to make anastomoses with a running fashion quickly and 
properly.

\section{Expert opinion 3: Dr. Raul Caso}

The standard anastomotic technique used for an open approach (interrupted sutures for the bronchial part and running suture for the membranous bronchus) is not as suitable for a minimally invasive approach. Whether performing a robotic approach, complete VATS, or hybrid VATS, the suture threads can get entangled which can be quite cumbersome. In addition, without the interrupted sutures being tied, this can result in suboptimal exposure of the anastomosis; this is of particular importance in minimally invasive thoracic surgery, in which surgeons have a limited field of view and narrow working space. Therefore, a running suture is preferred by most surgeons (for the bronchial and vascular anastomoses) because it simplifies the technical challenges of the anastomosis.

\section{Expert opinion 4: Dr. Giulio Maurizi}

End-to-end as well as conduit reconstruction of the pulmonary artery requires a running suture. Conversely, after a bronchial sleeve resection, an anastomosis made by interrupted suture may provide a better result, in particular in term of adjustment of caliber discrepancy between the two bronchial stamps. Sometimes, the employment of a partial running suture may be useful in the membranous portion of the anastomosis, or in the narrow mediastinal portion of the anastomosis when performing a lower bronchial sleeve.

\section{Discussion}

The most common indication for conversion to thoracotomy include endobronchial tumor presence requiring sleeve resection. The key to the success of the surgical procedure is patient selection. VATS SL was considered an absolute contraindication for centrally located tumors for many years (16,17). In 2002 (18), the first case of bronchial anastomosis SL was reported, but few case series have been published and experienced surgeons in the field of VATS have been slow to adopt and develop this technique $(16,17)$. The application of VATS SL is relatively limited because of challenges associated with the bronchial anastomosis $(19,20)$.

Compared with traditional thoracotomy, hybrid VATS had some advantages, for instance, the length of incision is smaller about $5-8 \mathrm{~cm}$ and the view is more direct visualization. Some reports showed the intercostal nerve damage causing post-thoracotomy pain for use of a rib retractor and rib spreading (21-23). A rib retractor is used without rib spreading in our hybrid VATS approach, the pain is theoretically less than traditional thoracotomy.

VATS approach is being applied to more complex procedures, such as SL, even and some authors have performed this procedure using uniportal VATS $(24,25)$. Surgeons do not directly access the tumor during the VATS procedure, especially for tumors invading the bronchus or vessels. In VATS, management of the vascular structure is technically challenging, especially the reconstruction of the bronchus and PA. Bronchial or vascular anastomosis by VATS is difficult because surgeons have a limited operative view and narrow working space. Currently, uniportal VATS SL performed only in a few centers because it requires considerable uniportal VATS experience and advanced training. Surgeons may face technical difficulties like bronchial or vascular anastomosis and intraoperative bleeding if lacking experience $(26,27)$. Obesity, severely attached diaphragm to the lower lobe, and enlarged fused lymph nodes usually make the uniportal VATS surgery extremely difficult because the bronchus and vascular structures are not readily accessible under these conditions (28).

Currently, robot-assisted thoracoscopic surgery (RATS) is applied in more complex surgical procedure including sleeve lobectomy. Cerfolio and colleagues (29) reported 8 patients underwent robotic simple sleeve lobectomy in 2016. Only a few cases of complex SL including double sleeve resection were performed under RATS. To our knowledge, Jiao and colleagues (30) reported the largest series of robotic sleeves about 67 cases, all the cases are bronchial sleeve without vessels reconstruction. Qiu and colleagues (31) publicated their sleeve resections experiences, the number of robotic sleeve resection cases is 49, most cases are simple sleeve lobectomy, the number of complex SL is small. Currently, RATS is not used for lung cancer treatment at most of hospitals in China because there is no DaVinci S equipment and higher cost for our health insurance uncovered. Therefore, the hybrid VATS is more economic benefit and it is easier to accept for surgeons in China compared to RATS.

Current literature shows that the hybrid VATS approach has advantages for selected NSCLC patients over conventional open surgery $(13,14)$. Dr. He et al. demonstrated the feasibility of hybrid VATS sleeve lobectomy for selected NSCLC patients with excellent long-term outcomes (15). Compared with their approach, our approach is more minimally invasive because it does 
not need to spread the rib, the direct vision is usually enough for bronchial sleeve resection even for vessels reconstruction or double sleeve resection. We introduce a hybrid VATS SL approach, which provides better-suturing angle, easier maneuverability, more ergonomic for the surgeons, thus making the anastomosis more reliable, safer, less challenging, while saving some time. The biggest technical challenges in sleeve resections are the anastomoses of the bronchus and PA. Okada et al. showed that the hybrid VATS approach was reasonable and satisfactory whenever a complete VATS approach was considered challenging, including a bronchoplasty (32). Twin-needle stitch and bi-directional continuous full-thickness suture have been used in most patients in our center. One hundred and thirty-five patients who underwent bronchial SL using this running suture technique have achieved satisfactory results in our institute. Literature has also shown that the simple continuous suture for the anastomosis of the bronchus is safe and reliable $(33,34)$. We favor the use of this hybrid VATS approach to perform the broncho-vascular anastomosis because it is easy to do and save much time. When encountering the high-caliber mismatch between the bronchus or PA, we contracted the more significant end by running suture with 4-0 prolene. Mahtabifard et al. have shown that there is no evidence that autologous tissue coverage in bronchial anastomosis procedure is necessary to prevent bronchial fistula (16). We performed bronchial anastomosis without autologous tissue coverage in our case. The postoperative bronchoscopy showed there was no sign of bronchial anastomotic stenosis or fistula, and the patient had an uneventful recovery. However, for double sleeve resection, we usually covered the bronchial anastomosis with mediastinal fat or pleural flap to avoid the postoperative disaster (bronchial fistula and possible massive bleeding after that).

A VATS approach can be utilized to perform bronchial SL in some centers. However, the hybrid VATS SL offers several advantages over complete VATS SL, including providing a better operative field, ensuring a safer and easier anastomosis, and saving some time, especially in patients with PA infiltration. As a result of these factors, the operation time is significantly reduced. In our experience, despite a slight increase in postoperative pain, patients tolerate it well and achieve a quick postoperative recovery. A hybrid VATS sleeve resection is more straightforward for most surgeons because it can provide an excellent operative field, ensure a safe surgical margin, and reduce operative time. It can be widely applied with intensive training and accumulation of surgical experience. Therefore, the hybrid VATS SL approach is safe and possible for treating locally advanced NSCLC if anatomical and oncological suitable.

\section{Conclusions}

The hybrid VATS SL provides an excellent operative field, a safe and easy anastomosis, and reduces operative time. It is suitable for complex SL cases especially in patients with PA infiltration. In summary, the hybrid VATS SL approach is safe and technical feasible for treating locally advanced NSCLC if anatomical and oncological suitable.

\section{Acknowledgments}

Funding: Supported by grants from Department of Liaoning Science and Technology, titled with The Construction of Liaoning Cancer Research Center (Lung Cancer) (1564992449013); Natural Science Foundation Guidance Program of Liaoning Province (2019-ZD-0589) ; Precise diagnosis and treatment and optimization of a clinical pathway for malignant tumor based on molecular markersthe research of precise treatment and optimization of clinical pathways for lung cancer (2019020176-JH1/10302); Technological Special Project of Liaoning Province of China (2019020176-JH1/103); Central financial fund for promoting medical service and safeguarding capability (Capability construction of medical and health organizations) - a subsidy to the Construction of Provincial Key Specialty; Research grant to introduced talents of Liaoning Cancer Hospital.

\section{Footnote}

Reporting Checklist: The authors have completed the CARE reporting checklist. Available at http://dx.doi.org/10.21037/ jtd-20-2679

Conflicts of Interest: All authors have completed the ICMJE uniform disclosure form and declare (available at http:// dx.doi.org/10.21037/jtd-20-2679). The authors have no conflicts of interest to declare.

Ethical Statement: The authors are accountable for all aspects of the work in ensuring that questions related to the accuracy or integrity of any part of the work are 
appropriately investigated and resolved. Written informed consent was obtained from the patient for publication of this manuscript and any accompanying images. All procedures performed in studies involving human participants were in accordance with the ethical standards of the institutional and/or national research committee(s) and with the Helsinki Declaration (as revised in 2013).

Open Access Statement: This is an Open Access article distributed in accordance with the Creative Commons Attribution-NonCommercial-NoDerivs 4.0 International License (CC BY-NC-ND 4.0), which permits the noncommercial replication and distribution of the article with the strict proviso that no changes or edits are made and the original work is properly cited (including links to both the formal publication through the relevant DOI and the license). See: https://creativecommons.org/licenses/by-nc-nd/4.0/.

\section{References}

1. Chen $\mathrm{W}$, Zheng R, Baade PD, et al. Cancer statistics in China, 2015. CA Cancer J Clin 2016;66:115-32.

2. Tseng TS, Gross T, Celestin MD, et al. Knowledge and attitudes towards low dose computed tomography lung cancer screening and smoking among African Americansa mixed method study. Transl Cancer Res 2019;8:S431-42.

3. Ma Z, Dong A, Fan J, et al. Does sleeve lobectomy concomitant with or without pulmonary artery reconstruction (double sleeve) have favorable results for non-small cell lung cancer compared with pneumonectomy? A meta-analysis. Eur J Cardiothorac Surg 2007;32:20-8.

4. Abdelsattar ZM, Shen R, Yendamuri S, et al. Outcomes After Sleeve Lung Resections Versus Pneumonectomy in the United States. Ann Thorac Surg 2017;104:1656-64.

5. Pagès $\mathrm{PB}$, Mordant $\mathrm{P}$, Renaud $\mathrm{S}$, et al. Sleeve lobectomy may provide better outcomes than pneumonectomy for non-small cell lung cancer. A decade in a nationwide study. J Thorac Cardiovasc Surg 2017;153:184-95.e3.

6. Park JS, Yang HC, Kim HK, et al. Sleeve lobectomy as an alternative procedure to pneumonectomy for non-small cell lung cancer. J Thorac Oncol 2010;5:517-20.

7. Balduyck B, Hendriks J, Lauwers P, et al. Quality of life after lung cancer surgery: a prospective pilot study comparing bronchial sleeve lobectomy with pneumonectomy. J Thorac Oncol 2008;3:604-8.

8. Mei J, Guo C, Xia L, et al. Long-term survival outcomes of video-assisted thoracic surgery lobectomy for stage
I-II non-small cell lung cancer are more favorable than thoracotomy: a propensity score-matched analysis from a high-volume center in China. Transl Lung Cancer Res 2019;8:155-66.

9. McKenna RJ Jr, Houck W, Fuller CB. Video-assisted thoracic surgery lobectomy: experience with 1,100 cases. Ann Thorac Surg 2006;81:421-5; discussion 425-6.

10. Bölükbas S, Baldes N, Eberlein M. Minimally invasive bronchial and bronchovascular sleeve resections. J Thorac Dis 2019;11:S1177-9.

11. Amer K. Video-assisted thoracic surgery double sleeve bilobectomy for lung cancer: The Sky is the Limit. J Thorac Dis 2019;11:6-7.

12. Soultanis KM, Chen Chao M, Chen J, et al. Technique and outcomes of 79 consecutive uniportal video-assisted sleeve lobectomies. Eur J Cardiothorac Surg 2019;56:876-82.

13. Jaus MO, Forcione A, Gonfiotti A, et al. Hybrid treatment of T3 chest wall lung cancer lobectomy. J Vis Surg 2018;4:32.

14. Nun AB, Simansky D, Rokah M, et al. Hybrid videoassisted and limited open (VALO) resection of superior sulcus tumors. Surg Today 2016;46:686-90.

15. He J, Shao W, Cao C, et al. Long-term outcome of hybrid surgical approach of video-assisted minithoracotomy sleeve lobectomy for non-small-cell lung cancer. Surg Endosc 2011;25:2509-15.

16. Mahtabifard A, Fuller CB, McKenna RJ Jr. Video-assisted thoracic surgery sleeve lobectomy: a case series. Ann Thorac Surg 2008;85:S729-32.

17. Li Y, Wang J. Video-assisted thoracoscopic surgery sleeve lobectomy with bronchoplasty. World J Surg 2013;37:1661-5.

18. Santambrogio L, Cioffi U, De Simone M, et al. Video assisted sleeve lobectomy for mucoepidermoid carcinoma of the left lower lobar bronchus: a case report. Chest 2002;121:635-6.

19. Predina JD, Kunkala M, Aliperti LA, et al. Sleeve lobectomy: current indications and future directions. Ann Thorac Cardiovasc Surg 2010;16:310-8.

20. Li Y, Wang J. Video-assisted thoracoscopic surgery sleeve lobectomy with bronchoplasty: an improved operative technique. Eur J Cardiothorac Surg 2013;44:1108-12.

21. Miyazaki T, Sakai T, Tsuchiya T, et al. Assessment and follow-up of intercostal nerve damage after video-assisted thoracic surgery. Eur J Cardiothorac Surg 2011;39:1033-9.

22. Rogers ML, Henderson L, Mahajan RP, et al. Preliminary findings in the neurophysiological assessment of intercostal nerve injury during thoracotomy. Eur J Cardiothorac Surg 
2002;21:298-301.

23. Swanson SJ, Herndon JE, 2nd, D'Amico TA, et al. Videoassisted thoracic surgery lobectomy: report of CALGB 39802--a prospective, multi-institution feasibility study. J Clin Oncol 2007;25:4993-7.

24. Gonzalez-Rivas D, Fernandez R, Fieira E, et al. Uniportal video-assisted thoracoscopic bronchial sleeve lobectomy: first report. J Thorac Cardiovasc Surg 2013;145:1676-7.

25. Gonzalez-Rivas D, Delgado M, Fieira E, et al. Left lower sleeve lobectomy by uniportal video-assisted thoracoscopic approach. Interact Cardiovasc Thorac Surg 2014;18:237-9.

26. Soultanis KM, Gonzalez-Rivas D. Uniportal video-assisted sleeve resections: how to deal with specific challenges. J Thorac Dis 2019;11:S1670-7.

27. Kocher GJ. Multiport and uniportal VATS sleeve resections. J Thorac Dis 2019;11:1091-3.

28. Guido Guerrero W, Gonzalez-Rivas D, Hernandez Arenas LA, et al. Techniques and difficulties dealing with hilar and interlobar benign lymphadenopathy in uniportal VATS. J Vis Surg 2016;2:23.

Cite this article as: Zhang $\mathrm{C}, \mathrm{Yu} \mathrm{Z,} \mathrm{Li} \mathrm{J,} \mathrm{Zu} \mathrm{P,} \mathrm{Yu} \mathrm{P,} \mathrm{Wang}$ G, Miyazaki T, Waseda R, Caso R, Maurizi G, Liu H. Hybrid video-assisted thoracoscopic surgery sleeve lobectomy for non-small cell lung cancer: a case report. J Thorac Dis 2020;12(11):6836-6846. doi: 10.21037/jtd-20-2679
29. Cerfolio RJ. Robotic sleeve lobectomy: technical details and early results. J Thorac Dis 2016;8:S223-6.

30. Jiao W, Zhao Y, Qiu T, et al. Robotic Bronchial Sleeve Lobectomy for Central Lung Tumors: Technique and Outcome. Ann Thorac Surg 2019;108:211-8.

31. Qiu T, Zhao Y, Xuan Y, et al. Robotic sleeve lobectomy for centrally located non-small cell lung cancer: A propensity score-weighted comparison with thoracoscopic and open surgery. J Thorac Cardiovasc Surg 2020;160:838-46.e2.

32. Okada M, Sakamoto T, Yuki T, et al. Hybrid surgical approach of video-assisted minithoracotomy for lung cancer: significance of direct visualization on quality of surgery. Chest 2005;128:2696-701.

33. Aigner C, Jaksch P, Seebacher G, et al. Single running suture--the new standard technique for bronchial anastomoses in lung transplantation. Eur J Cardiothorac Surg 2003;23:488-93.

34. Huang J, Li J, Qiu Y, et al. Thoracoscopic double sleeve lobectomy in 13 patients: a series report from multicenters. J Thorac Dis 2015;7:834-42. 